"Fl suber ile mis hiipses

Año 13.

Núm. 33

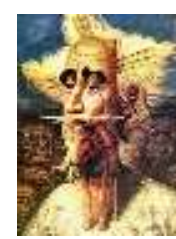

Revista de Investigación

Académica sin Frontera

ISSN: 2007-8870

http://revistainvestigacionacademicasinfrontera.com

Recibido el 2 de septiembre de 2020. Dictaminado mediante arbitraje favorablemente 24 de septiembre 2020

\title{
Clima organizacional y el programa escuela de tiempo completo, en una comunidad rural del sureste mexicano
}

\section{Organizational climate and full time school program in a rural community in the southeast of Mexico}

Martha Patricia Díaz Cruz, Universidad Juárez Autónoma de Tabasco, http://orcid.org/0000-0002-8376-7172, mpatydiaz@hotmail.com

Aída Dinorah García Álvarez, Universidad Juárez Autónoma de Tabasco, http://orcid.org/0000-0002-2846-6924, aida.garcia@ujat.mx

\section{Resumen}

Este documento describe los resultados obtenidos de una investigación realizada en una escuela de educación básica en el sureste de México, incluida en un programa de apoyo para la educación de calidad del gobierno federal. El objetivo de la investigación fue conocer el clima organizacional, para identificar factores de motivación y liderazgo que influyen en el mismo. El estudio fue de tipo descriptivo con enfoque mixto. La población examinada fue el personal que integra la plantilla laboral de la institución seleccionada. Los datos fueron recolectados mediante un cuestionario aplicado, distribuido en tres apartados: aspectos socio demográficos, percepción sobre motivación y liderazgo. Los principales hallazgos revelaron que el nivel de motivación del personal docente es bajo, el tipo de liderazgo de la escuela no favorece el funcionamiento apropiado de la 
"Fl suber ise mis hijics

Año 13.

Núm. 33

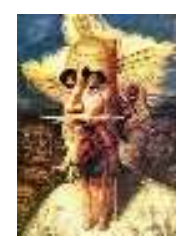

Revista de Investigación

Académica sin Frontera

ISSN: 2007-8870

\section{http://revistainvestigacionacademicasinfrontera.com}

Recibido el 2 de septiembre de 2020. Dictaminado mediante arbitraje favorablemente 24 de septiembre 2020 institución, además los profesores no perciben un ambiente motivador para desempeñar las actividades añadidas por el Programa de Escuelas de Tiempo Completo. Coinciden en que el ambiente laboral es tenso. El principal motor que los impulsa a emprender sus labores es el compromiso con los estudiantes.

Palabras claves: Clima Organizacional, Liderazgo, Motivación, Escuela de tiempo completo.

\section{Abstract}

The purpose of this document describes the results achieved from a study of the organizational climate in an elementary school in the Southeast of Mexico; it is included in a federal support educational program. The aim of the research was to get an appreciation of the organizational climate to identify the motivational factors and kind of leadership. It was a descriptive study with a mixed approach. The sample examined was the total teaching staff.

The data was collected through a questionnaire, distributed in three sections: sociodemographic aspects, and perception of motivation and leadership. The findings revealed a low motivation level and the kind of leadership has affected the well-functioning of school. Furthermore, the professors do not perceive a motivating environment to perform the activities defined by the Full Time School Program. They agree that labor environment is tense. The commitment to student is the main driving force to accomplish their activities.

Keywords: Organizational climate, Leadership, Motivation, Full time school 
"Fl suler ide mis hiips

Año 13.

Núm. 33

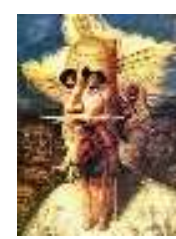

Revista de Investigación

Académica sin Frontera

ISSN: 2007-8870

\section{http://revistainvestigacionacademicasinfrontera.com}

Recibido el 2 de septiembre de 2020. Dictaminado mediante arbitraje favorablemente 24 de septiembre 2020

\section{Introducción}

El sistema educativo mexicano se encuentra en proceso de fuertes cambios, debido fundamentalmente a las reformas educativas de reciente implementación, aunada a otras reformas estructurales como la laboral y la fiscal que buscan elevar indicadores de calidad de vida en la población. Debido a las nuevas propuestas los trabajadores de la educación muestran resistencia a través de diversos movimientos de protesta, lo que evidencia desacuerdos e inconformidad, este mismo rechazo se refleja en el ambiente escolar, lo que afecta el desempeño de las actividades académicas y con ello a los estudiantes. Por ello, además de establecer nuevos modelos educativos, nuevas reglas de ingreso al servicio docente; es imperativo que las instituciones educativas reconozcan la importancia del conocimiento preciso del ambiente y las condiciones de trabajo en las escuelas. El conocimiento preciso de los factores que motivan el trabajo, no solo del personal docente, sino de todos los actores de la comunidad educativa, contribuye en el diseño de estrategias de mejoramiento y toma decisiones que garanticen el cumplimiento de objetivos y metas para elevar la calidad de la educación.

El estudio que aquí se presenta centra su análisis en la relación entre, el clima organizacional y los factores de motivación en el personal que labora en la escuela primaria Miguel Hidalgo y Costilla, esta es apoyada por el programa: Escuelas de Tiempo Completo; creado por el Gobierno Federal y cuya finalidad es generar ambientes de trabajo, propicios para mejorar la calidad de la enseñanza. La intención es lograr un conocimiento más amplio de los factores que influyen en el ambiente de trabajo y conseguir información más detallada.

La escuela Miguel Hidalgo y Costilla se encuentra en crecimiento de la matrícula estudiantil; el programa que actualmente la beneficia ha permitido que la población de alumnado incremente. Sin embargo, las características de la institución, la forma en que 
"Flis suber ide mis hiipses

Año 13.

Núm. 33

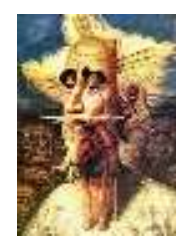

Revista de Investigación

Académica sin Frontera

ISSN: 2007-8870

http://revistainvestigacionacademicasinfrontera.com

Recibido el 2 de septiembre de 2020. Dictaminado mediante arbitraje favorablemente 24 de septiembre 2020

está estructurada, el tipo de liderazgo y la motivación hacia los docentes afectan el proceso enseñanza - aprendizaje.

Se utilizan para esta investigación, dos instrumentos validados: una encuesta de clima organizacional y una entrevista, basados en la hipótesis: La motivación y liderazgo son factores del clima organizacional, que influyen el grado de desempeño del personal docente que labora en la escuela primaria Miguel Hidalgo y Costilla del municipio de Centro Tabasco.

Lo relevante de este presente estudio se encuentra en la importancia que reviste conocer con certeza la situación del Clima Organizacional, los factores que motivan al personal y el tipo de liderazgo que se ejerce, a fin de conocer factores que pueden afectar el cumplimiento de los objetivos institucionales.

\section{Revisión de la literatura}

A partir de la revisión de los estudios realizados sobre lima Organizacional se entiende a este como las características del ambiente de trabajo de una institución ya sea positiva 0 negativa. La importancia de indagar sobre diversos factores que de alguna manera impactan a las instituciones educativas dentro del territorio mexicano, se torna cada vez más motivador y necesario, debido a que, en los últimos años se han implementado diversos programas educativos cuyos resultados ya deben saltar a la vista.

La educación básica en México se compone de tres niveles: preescolar, primaria y secundaria. De acuerdo con el censo realizado en 2013, por la Secretaría de Educación Pública (SEP), el país cuenta con 273,317 incluidos los planteles de educación especial (ver Figura 1). 
"Fl solber ile mis hiips

Año 13.

Núm. 33

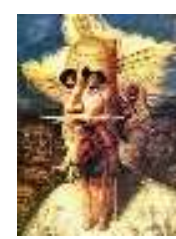

Revista de Investigación Académica sin Frontera

ISSN: 2007-8870

\section{http://revistainvestigacionacademicasinfrontera.com}

Recibido el 2 de septiembre de 2020. Dictaminado mediante arbitraje favorablemente 24 de septiembre 2020

\begin{tabular}{|c|c|c|}
\hline Centros de trabajo visitados & Absolutos & \% \\
\hline Centros de trabajo planeados para el operativo: & 270609 & $99.0 \%$ \\
\hline Altas aprobadas por los comités (nuevos centros): & 2708 & $1.0 \%$ \\
\hline Total: & 273317 & $100 \%$ \\
\hline Centros no activos durante el ciclo escolar 2013-2014 & 2094 & $0.8 \%$ \\
\hline Bajas dictaminadas por los comités: & 9569 & $3.7 \%$ \\
\hline Total de centros en operación durante el ciclo escolar 2013-2014 & 261654 & \\
\hline
\end{tabular}

Figura 1. Cobertura de centros de trabajo de la Secretaría de Educación Pública. Fuente: SEP (2013).

La población total de alumnos fue de 23, 667,973 (ver Figura 2). Cada uno de estos planteles con características similares como son, alumnos menores de 15 años, personal docente y administrativo, padres de familias y la infraestructura de la institución en la que se desenvuelve la comunidad estudiantil, como principales actores del proceso educativo.

\begin{tabular}{|l|c|c|}
\hline \multicolumn{3}{|c|}{ Alumnos } \\
\hline & Absoluto & Porcentaje \\
\hline $\begin{array}{l}\text { Alumnos en centros de trabajo } \\
\text { censados }\end{array}$ & 23667973 & 92.1 \\
\hline $\begin{array}{l}\text { Alumnos en centros no } \\
\text { censados (cifras de planeación)* }\end{array}$ & 2018156 & 7.9 \\
\hline Total & $\mathbf{2 5 6 8 6 1 2 9}$ & 100 \\
\hline
\end{tabular}


"Fl suler ide mis hiipses

Año 13.

Núm. 33

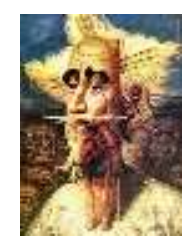

Revista de Investigación

Académica sin Frontera

ISSN: 2007-8870

\section{http://revistainvestigacionacademicasinfrontera.com}

Recibido el 2 de septiembre de 2020. Dictaminado mediante arbitraje favorablemente 24 de septiembre 2020

Figura 2. Alumnos censados por la Secretaría de Educación Pública. Fuente: SEP (2013).

El gobierno mexicano en su lucha por fortalecer la calidad de la educación, según la reforma hecha al artículo 3 de la Constitución Política de Los Estados Unidos Mexicanos ha implementado diversos programas como estrategias educativas para mejorar el proceso de enseñanza - aprendizaje del alumno en educación básica. Es así como surge el programa de Escuelas de Tiempo Completo según el acuerdo publicado en el diario oficial de la federación el 21 de diciembre del 2014, con la finalidad de crear condiciones institucionales para que los alumnos tengan mayores y mejores oportunidades de alcanzar los aprendizajes y desarrollar las competencias definidas en los planes y programas de estudio vigentes. De este modo se instaura un aumento de horas al servicio educativo en las Escuelas de Tiempo Completo. Así se aumenta cincuenta por ciento de horas el servicio educativo en las Escuelas de Jornada Ampliada, de manera que un alumno que curse los seis grados de educación primaria en dicha institución, habrá tomado tres ciclos escolares más de clases que los de una escuela regular y, uno de preescolar que curse tres grados del nivel, habrá tomado un ciclo y medio más que los de planteles con jornada tradicional. Lo anterior busca permitir a los profesores fortalecer el trabajo de los contenidos curriculares, intensificar el desarrollo de la competencia lectora y matemática, la comprensión de la ciencia, incorporar la adquisición del inglés como segunda lengua, desarrollar habilidades para el uso educativo de las Tecnología de la Información y la Comunicación (TIC's), fomentar una cultura para una vida saludable y fortalecer las competencias para la convivencia y desarrollo cultural (SEP, 2014).

La relevancia de conocer los diversos factores que afectan a las instituciones educativas en México cobra cada vez mayor importancia, ya que lograr estándares de calidad no solo depende de ciertos actores del proceso educativo, sino que como toda organización necesita mirar hacia el interior, prestar atención a un factor un tanto descuidado como es 
"Fl suleer ide mis hiips

Año 13.

Núm. 33

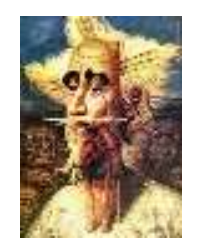

Revista de Investigación

Académica sin Frontera

ISSN: 2007-8870

\section{http://revistainvestigacionacademicasinfrontera.com}

Recibido el 2 de septiembre de 2020. Dictaminado mediante arbitraje favorablemente 24 de septiembre 2020

el ambiente de trabajo. Este fenómeno requiere ser comprendido y evaluado para implementar acciones de mejora en el afán de contar con ambientes propicios para el desempeño óptimo de las funciones. Por ello el presente estudio abordó el clima organizacional de una institución educativa.

En seguida se revisan aspectos teóricos relacionados con el estudio del clima organizacional, este se entiende como el ambiente en el que interactúa el personal de una agrupación para desempeñar las actividades correspondientes.

Una de las definiciones más recurridas en este ámbito es la de Chiavenato (2002) que define el clima organizacional como la calidad del ambiente de la organización que es percibido o experimentado por sus miembros y que influye ostensiblemente en su comportamiento. El clima organizacional entraña un cuadro amplio y flexible de la influencia del ambiente en la motivación. Por consiguiente, el clima organizacional se refiere a las características del medio ambiente de trabajo, las cuales son percibidas directa o indirectamente por los trabajadores que se desempeñan en ese ambiente, y repercute en el comportamiento laboral. Es favorable cuando satisface las necesidades personales de los participantes y eleva su estado de ánimo. Es desfavorable cuando despierta la frustración por no satisfacer esas necesidades.

En este caso una institución educativa requiere de un ambiente o atmósfera laboral en que los docentes, personal administrativo y de apoyo confluyan de manera positiva ya que este a su vez repercute en los estudiantes. Diversos estudios revisados señalan que los factores del clima organizacional son aquellos que el personal o empleados perciben como aspectos determinantes de la calidad de vida en el trabajo de una organización determinada (Arias, 2006). Es por ello que se han desarrollado investigaciones sobre el clima organizacional en instituciones educativas de México. Al respecto, Lamoyi (2007) realizó un estudio de clima organizacional en las escuelas secundarias generales y técnicas del estado de 
"Fl suler ide mis hiips

Año 13.

Núm. 33

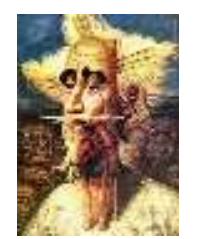

Revista de Investigación

Académica sin Frontera

ISSN: 2007-8870

\section{http://revistainvestigacionacademicasinfrontera.com}

Recibido el 2 de septiembre de 2020. Dictaminado mediante arbitraje favorablemente 24 de septiembre 2020 Tabasco, a través de las dimensiones de creencias, sentido de comunidad y liderazgo directivo con la finalidad de conocer el grado de integración social en cada una de ellas y en su conjunto. Aunque el clima organizacional es un tema extenso y controversial en la actualidad, este tiene sus orígenes en la mente de gran cantidad de investigadores que han realizado diversos estudios al respecto y que de una $u$ otra forma han obtenido resultados, basándose en componentes como: la motivación y el liderazgo.

Arce, A. (2017) describe el clima organizacional como la percepción que tienen los miembros de una organización de su funcionamiento estructural y dinámico, esta percepción influye en los niveles de satisfacción y motivación en la disposición a permanecer en la organización y en el desempeño del personal. El autor destaca que la definición de la estructura, y como esta se mueve en función de la ejecución de las tareas se refleja en el desempeño de los miembros. Desde luego, dicha percepción se traduce en acciones concretas de los individuos, que a su vez impactan el cumplimiento de los objetivos de la organización. Por ello, la importancia que reviste el estudio del factor humano y aquellas incidencias que determinan su bienestar o inconformidad en el medio laboral.

Medina, A. (2017) afirma que la percepción es un elemento en el que convergen variedad de autores al definir el clima organizacional. Esta percepción generalmente gira en torno a: estructura organizativa, estilos de dirección o liderazgo y los mecanismos de recompensa o reconocimiento, los mismos que inciden en su desempeño, puesto que una persona desarrollará mejor su trabajo si se encuentra bien consigo misma y si está a gusto con su entorno laboral.

Al respecto Abraham Maslow (1943) es uno de los teóricos que intenta explicar la motivación a través de su teoría de las necesidades fisiológicas, en la cual el ser humano centra sus criterios para desempeñarse durante la vida. Este estudio parte 
"Fl suler ide mis hiips

Año 13.

Núm. 33

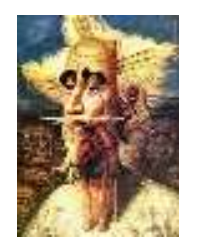

Revista de Investigación

Académica sin Frontera

ISSN: 2007-8870

\section{http://revistainvestigacionacademicasinfrontera.com}

Recibido el 2 de septiembre de 2020. Dictaminado mediante arbitraje favorablemente 24 de septiembre 2020 de la idea que el clima organizacional ejerce influencia en el proceso de aprendizaje y en la relación que debe existir entre el personal o miembros de la institución u organización. Los factores que impactan el clima o ambiente organizacional son muy variados, a la vez que determinan también el rendimiento laboral. De acuerdo con Van der Westhuizen y Mentz, (2007), estos aspectos se dividen en dos categorías denominadas: factores sistémicos y factores personales 0 individuales. Los sistémicos se refieren entre otros, a la cultura organizacional y la salud organizacional mientras que los individuales o personales abarcan, la motivación laboral, la satisfacción laboral, estrés laboral y desempeño laboral.

Para este estudio se abordaron dos dimensiones: la motivación y el liderazgo con el fin de conocer la influencia que ejercen en el clima organizacional de la institución en cuestión.

La motivación juega un papel preponderante dentro de una institución educativa, debido a que es uno de los motores para desenvolverse en cualquier ámbito que se le presente al ser humano, sin motivación cualquier objetivo planteado es probable que no llegue a concretarse.

En ese sentido, Amorós (2007) menciona que la motivación se refiere a las fuerzas que actúan sobre un individuo o en su interior y originan, que su comportamiento sea de una forma determinada, dirigida hacia las metas condicionadas por la capacidad del esfuerzo de satisfacer alguna necesidad individual.

De igual forma el liderazgo también juega un papel central en una determinada empresa puesto que es el líder quien dirige hacia el logro de metas. Los líderes deben tener la capacidad de planear y de dirigir una institución, pero el papel central de un líder es influir en los demás para de esta manera considerarse un líder con capacidad de mando.

Según Montalván, C. (1999), la palabra liderazgo proviene del origen inglés y su significado es: conducción, guía, influencia, autoridad. Lo define como una función que 
"Fl suler ite mis hijisa

Año 13.

Núm. 33

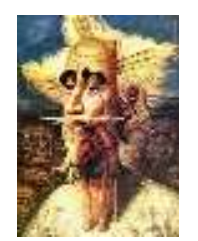

Revista de Investigación

Académica sin Frontera

ISSN: 2007-8870

\section{http://revistainvestigacionacademicasinfrontera.com}

Recibido el 2 de septiembre de 2020. Dictaminado mediante arbitraje favorablemente 24 de septiembre 2020 realizan algunas personas que ejercen autoridad sobre otra. En este sentido, Martínez (2003) considera al liderazgo como el proceso de influir en las actividades que realiza una persona o grupo para la consecución de una meta. En toda organización o grupo de trabajo se genera un ambiente que propicia cierta forma de llevar a cabo las tareas, a su vez este ambiente impactará el logro de los objetivos y metas establecidos por la organización.

De manera general, se conoce gran variedad de tipos de liderazgo, a continuación, se describen algunos de ellos:

El Autocrático: se muestra eficiente, asume responsabilidades; impone órdenes y espera la ejecución de las mismas. Se apoya en sistemas legales, decide las actividades y busca garantizar que los trabajadores lleven a cabo las labores por medio de amenazas y castigos.

El Paternalista: Representa una variante del tipo de liderazgo autoritario. Es indulgente y aparenta interés por el bienestar de los demás. Justifica todas las acciones. Este tipo de liderazgo es frecuente en las empresas de corte familiar.

El Permisivo: tipo de liderazgo que evita usar el poder y responsabilidad. El líder no se preocupa por lo que sucede, no ejerce autoridad sobre los trabajadores para cumplir las labores. Predomina un exceso de confianza. Otorga a los subordinados un alto grado de independencia operativa. En la práctica no funciona puesto que propicia el relajamiento, la indisciplina y la baja productividad.

El Democrático: Este tipo de liderazgo se caracteriza por diseminar la autoridad y funciona en determinadas organizaciones. Procura un sistema de igualdad de derechos y de deberes. Existe participación de los trabajadores en el proceso de toma de decisiones por lo que el líder y el grupo actúa como una unidad social.

El Situacional: Es el liderazgo más adecuado, efectivo y conveniente para una determinada organización. La principal característica es que se ejerce de acuerdo con 
"Fl suleer ide mis hiips

Año 13.

Núm. 33

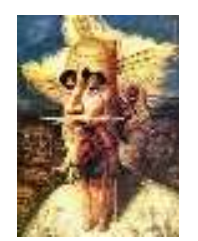

Revista de Investigación

Académica sin Frontera

ISSN: 2007-8870

\section{http://revistainvestigacionacademicasinfrontera.com}

Recibido el 2 de septiembre de 2020. Dictaminado mediante arbitraje favorablemente 24 de septiembre 2020 las circunstancias que se presentan, requiere de una gran capacidad de adaptación y un excelente manejo de recursos personales, para el ejercicio de la autoridad. Su sustento es de orden práctico.

Los directivos o puestos de influencia en una organización pueden adoptar varios tipos de liderazgo. En definitiva, un buen liderazgo es indispensable para el logro de los objetivos previstos. Los dirigentes deben ser capaces de planear y organizar. Así el éxito depende del comportamiento, habilidades, acciones apropiadas y no de las características personales de cada directivo, las cuales son fijas.

En el contexto educativo para sostener un liderazgo en el aula, los docentes requieren desarrollar las actividades como eje del cambio educativo y asegurar mejores aprendizajes para los niños y niñas, además de contar con la gestión escolar e impulso del liderazgo de los directores de la institución. Ya que las reformas educativas rara vez tienen éxito sin un liderazgo efectivo, tanto a nivel de sistema como de cada escuela (Vélaz de Medrano y Vaillant, 2009).

Las definiciones anteriores destacan la importancia y la forma en que las personas deberían desempeñar las funciones laborales como miembros de una organización para la consecución de metas. En relación a ello, los estudios enfocados en el clima organizacional coinciden en su mayoría en que, este es principalmente influenciado por el rol que desempeñan los diferentes miembros en la organización. Según Van der Westhuizen y Mentz (2007) en la gestación del clima o atmósfera organizacional predominan principalmente dos factores: el primero es la conducta de los líderes en la escuela, el comportamiento, en especial del director o directora influye en la manera en que el personal experimenta su manera de dirigir. El modo de conducir las actividades y funciones de la escuela, afectan la forma en que el personal experimenta las actividades y por ende influye en el clima laboral de la institución. El segundo factor es el comportamiento del personal. Las disposiciones generales con las que el personal 
"Fl suler de mis hijics

Año 13.

Núm. 33

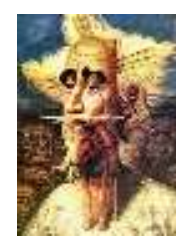

Revista de Investigación

Académica sin Frontera

ISSN: 2007-8870

\section{http://revistainvestigacionacademicasinfrontera.com}

Recibido el 2 de septiembre de 2020. Dictaminado mediante arbitraje favorablemente 24 de septiembre 2020

desempeña sus labores y la aceptación del liderazgo de la escuela influyen en la forma en que el personal impacta su ambiente laboral.

En este contexto es necesario entonces profundizar en el conocimiento del ambiente que se genera al interior de las instituciones educativas y determinar los factores que mayormente impactan el desempeño del personal y por tanto el logro de las metas $u$ objetivos para una educación de calidad. Gran parte del éxito de los programas dirigidos para tal fin depende de las condiciones del clima laboral en que los actores del proceso educativo llevan a cabo sus tareas.

\section{Metodología}

Con el fin de lograr mayor acercamiento al contexto natural del fenómeno de estudio la investigación se abordó desde un enfoque mixto, para además de la medición de variables, conocer más ampliamente los puntos de vista de los sujetos de estudio. Según Lincoln y Guba (2000), los enfoques cuantitativos y cualitativos nos proporcionan una visión o fotografía o trozo de la realidad. El tipo de diseño del estudio es una investigación no experimental ya que no se manipula ninguna variable, sino que se estudia el contexto natural del clima organizacional centrado en el liderazgo y ambiente motivacional en que se desenvuelven los docentes de la institución educativa seleccionada. Es de corte transversal y descriptivo puesto que los datos fueron recolectados en un solo momento en el tiempo, con la finalidad de describir las variables determinadas con anterioridad. El carácter descriptivo comprende la descripción, registro, análisis e interpretación de la naturaleza de los datos, que revelan a su vez la naturaleza del fenómeno ya que se busca especificar las propiedades y las características importantes del Clima organizacional de la escuela primaria Miguel Hidalgo y Costilla de la ciudad de Villahermosa Tabasco beneficiada con el programa escuela de tiempo completo. 
"Fl sulber ide mis hijise

Año 13.

Núm. 33

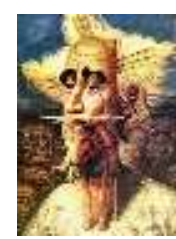

Revista de Investigación

Académica sin Frontera

ISSN: 2007-8870

\section{http://revistainvestigacionacademicasinfrontera.com}

Recibido el 2 de septiembre de 2020. Dictaminado mediante arbitraje favorablemente 24 de septiembre 2020 Los datos cuantitativos se recolectaron a través de un cuestionario ajustado al presente estudio donde se tomó como referencia la investigación de Lamoyi (2007), este consta de 31 reactivos, distribuidos en tres apartados: el primero aborda aspectos sociodemográficos, el segundo apartado se refieren a preguntas a escala de Likert referentes a la motivación y el liderazgo que se percibe por parte del personal docente de la institución seleccionada. Se administró a las 12 personas que componen la planta docente, por tanto, el $100 \%$ de la población funge como objeto de estudio ya que esta es reducida. El instrumento se distribuyó de manera libre y anónima. Los datos se procesaron con el programa SPSS.

En la Tabla 1 se muestra la descripción de las variables abordadas.

Tabla 1. Descripción de variable

\begin{tabular}{|c|c|c|c|}
\hline Variable & Dimensión & Definición & $\begin{array}{c}\text { Ítems del } \\
\text { cuestionario }\end{array}$ \\
\hline Sociodemográfica & $\begin{array}{l}\text { Edad } \\
\text { Sexo } \\
\text { Escolaridad } \\
\text { Tipo de plaza }\end{array}$ & $\begin{array}{l}\text { Tiempo que ha vivido el } \\
\text { entrevistado. } \\
\text { Condición orgánica masculina o } \\
\text { femenina. } \\
\text { Último grado escolar cursado y } \\
\text { concluido. } \\
\text { Tipo de contratación que posee } \\
\text { actualmente. }\end{array}$ & $1-7$ \\
\hline
\end{tabular}


"Fl suler ide mis hiipses

Año 13.

Núm. 33

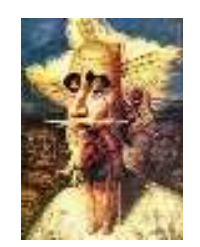

Revista de Investigación

Académica sin Frontera

ISSN: 2007-8870

\section{http://revistainvestigacionacademicasinfrontera.com}

Recibido el 2 de septiembre de 2020. Dictaminado mediante arbitraje favorablemente 24 de septiembre 2020

\begin{tabular}{l|l|l|l}
\hline & Liderazgo & $\begin{array}{l}\text { El proceso de dirigir e influir en las } \\
\text { actividades laborales de los } \\
\text { miembros de un grupo }\end{array}$ & $1-15$ \\
organizacional & Motivación & $\begin{array}{l}\text { Factores que logran una } \\
\text { determinada conducta en los } \\
\text { miembros de una institución. }\end{array}$ & $16-31$
\end{tabular}

Nota: En esta Tabla describe las variables e ítems utilizados en la investigación. La primera columna muestra las variables utilizadas, en la segunda columna se encuentran la dimensión de la variable, en la tercera columna se encuentra el indicador y en la última columna están los ítems del cuestionario.

Fuente: Elaboración propia

Con la finalidad de profundizar en el estudio, acercarse al contexto natural y obtener información detallada de la forma en que los sujetos de estudio experimentan o transitan en el ambiente de trabajo, se abordó también el estudio desde un enfoque cualitativo. De acuerdo con (Hernández, Fernández y Baptista, 2010) los estudios cualitativos se definen como un conjunto de prácticas interpretativas que hacen al mundo visible, lo transforman y convierten en una serie de representaciones en forma de observaciones, anotaciones, grabaciones y documentos. Es naturalista (porque estudia a los objetos y seres vivos en sus contextos o ambientes naturales) e interpretativo (pues intenta encontrar sentido a los fenómenos en términos de los significados que las personas les otorguen). Este se basa en métodos de recolección de datos no estandarizados, no emplea mediciones numéricas, la recolección de los datos consiste en obtener las perspectivas y puntos de vista de los participantes. En este caso, los datos cualitativos se recolectaron con una entrevista estructurada para conocer los puntos de vista del director y los docentes. La 
"Fl suleer ile mis hiips

Año 13.

Núm. 33

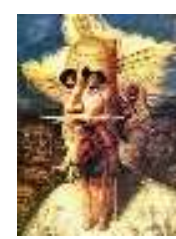

Revista de Investigación

Académica sin Frontera

ISSN: 2007-8870

\section{http://revistainvestigacionacademicasinfrontera.com}

Recibido el 2 de septiembre de 2020. Dictaminado mediante arbitraje favorablemente 24 de septiembre 2020 entrevista cualitativa se define como una reunión para intercambiar información entre el entrevistado y el entrevistador.

\section{Resultados}

Los datos derivados de la aplicación del cuestionario mostraron que los sujetos de estudio tienen bien claro los acuerdos y las metas que deben cumplir con el programa escuela de tiempo completo como lo exige la institución. Además, manifiestan que existe esfuerzo colaborativo por parte de cada uno de ellos, para dar lo mejor de sí mismo en sus espacios áulicos, producto del esmero y dedicación, a pesar de no contar con apoyo administrativo en sus actividades escolares ni la adecuada infraestructura; debido a la falta de espacio suficiente para los grupos de alumnos que tienen que tomar clases al aire libre o en lugares donde el clima favorezca.

Respecto a la valoración del esfuerzo y tiempo dedicado a sus respectivas actividades, el $100 \%$ de los participantes consideró que no es una pérdida de tiempo hacer lo mejor como profesores en esta escuela, a su vez hacen un esfuerzo colectivo para que los alumnos aprendan y de esta manera cumplir con los objetivos de la organización. La mayoría, aunque les gusta su trabajo, no realizan las actividades con entusiasmo, pero esto no impide que busquen estrategias de enseñanza para alumnos con bajo rendimiento escolar. El hecho de verse afectados por la organización y el poco esmero de las autoridades resta compromiso con las actividades fuera de clase, ya que de ninguna manera serán objeto de reconocimiento y apoyo por parte de sus líderes y de los padres y/o tutores de familia. Estas respuestas indican que la escuela carece de un clima organizacional que estimule el cumplimiento de las actividades y trae como resultado la falta de motivación del personal que labora dentro de la institución.

En relación al nivel de motivación del personal docente, los hallazgos muestran que el nivel de motivación es bajo, debido a que no cuentan con estímulos suficientes que les 
"Fl suleer ide mis hiips

Año 13.

Núm. 33

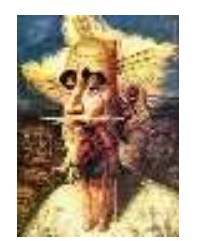

Revista de Investigación

Académica sin Frontera

ISSN: 2007-8870

\section{http://revistainvestigacionacademicasinfrontera.com}

Recibido el 2 de septiembre de 2020. Dictaminado mediante arbitraje favorablemente 24 de septiembre 2020 motive en el trabajo, sin embargo, reconocen y están consciente que la población estudiantil no es responsable del comportamiento de la administración de la organización y esto los mueve a realizar el trabajo de la mejor manera posible.

En lo concerniente al tipo de liderazgo que se presenta en la institución y el impacto que tiene en el clima organizacional, se identificó qué el tipo de liderazgo de la escuela no favorece el buen funcionamiento de la institución, ya que el director es un docente frente a grupo, que al mismo tiempo desempeña el cargo de director. Los docentes visualizan esta dirección como una imposición por parte de las autoridades educativas, puesto que no cumple con el tiempo suficiente para desempeñar las tareas de director. Este debe realizar ambas funciones en un mismo horario, esto genera que las gestiones sean poco eficaces o nulas. Por lo tanto, no se ha alcanzado una institución de vanguardia, con las condiciones que una escuela de tiempo completo requiere para cumplir cabalmente con los objetivos institucionales. El liderazgo que se manifiesta en esta organización educativa tiene rasgos del tipo permisivo y al igual que del autocrático, el director asume un rol de portavoz de las autoridades educativas superiores, transmite órdenes, dicta las actividades y espera el cumplimiento de las mismas. Sin embargo, no asume la responsabilidad, del cumplimiento de objetivos y metas. Concede a los docentes libertad en actividades añadidas por la extensión de horario según el Programa de Escuelas de Tiempo Completo. La doble función, en este caso, disminuye probablemente la dedicación a la planeación y supervisión de las actividades.

Una práctica frecuente en el sistema educativo mexicano es la imposición de directivos, no suelen ser seleccionados mediante concurso o examen como lo indican las normas vigentes. Además, no disponen del tiempo suficiente para desempeñar ambos roles, el de docente y administrador a la vez. 
"Fl suler ide mis hiips

Año 13.

Núm. 33

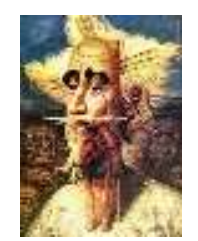

Revista de Investigación

Académica sin Frontera

ISSN: 2007-8870

\section{http://revistainvestigacionacademicasinfrontera.com}

Recibido el 2 de septiembre de 2020. Dictaminado mediante arbitraje favorablemente 24 de septiembre 2020

En lo concerniente a los datos cualitativos recaudados, el director expresó inconformidad acerca de la eficacia de su labor y ratifica lo difícil que es desempeñar dos funciones (docente y directivo) al mismo tiempo, puesto que ambas son demandantes.

Respecto al estado de la relación con sus subordinados, admite que la doble función en la escuela, demerita alguna de ellas. Sobre todo, descuida actividades administrativas, lo que ocasiona exigencias y presiones del personal docente, lo que genera un ambiente tenso.

En cuanto a la resolución de los conflictos que se presentan en la escuela; aseveró que la mayor parte de los conflictos son externos, con los padres de familia pero que trata de apoyar a sus compañeros docentes de la mejor forma. Además, reconoce poca efectividad en las gestiones de infraestructura pues son múltiples las actividades que desempeñar y este rubro ha sido descuidado.

Un aspecto muy sensible para los docentes bajo su dirección es el reconocimiento al desempeño de sus labores, al respecto, asegura que no depende de él otorgar recompensa por desempeño lo cual corresponde a las autoridades superiores.

Los docentes no perciben acciones motivadoras para desempeñar las actividades añadidas por el Programa de Escuelas de Tiempo Completo, sin embargo, los errores si son puntualmente señalados. También coinciden en que, el ambiente laboral es tenso y los espacios no son adecuados para desempeñar su trabajo en un horario extendido.

A pesar de las circunstancias, el personal entrevistado sostiene que emprenden sus labores con vigor y entusiasmo, sobre todo por sus alumnos ya que, aunque no cuenten con los recursos, siempre tratan de dar lo mejor de sí mismos. Aunque el desánimo surge del agobio por la cantidad de lineamientos de la reforma educativa, consideran como desfavorables para el buen desempeño de sus labores. La motivación por parte de su jefe directo es casi nula, cada parte hace su trabajo y cada uno trata de hacer lo mejor que puede y aunque el jefe trata de apoyar, existen limitaciones, ya que la jerarquía es 


\section{Revista de Investigación}

Año 13.

Académica sin Frontera

Núm. 33

ISSN: 2007-8870

\section{http://revistainvestigacionacademicasinfrontera.com}

Recibido el 2 de septiembre de 2020. Dictaminado mediante arbitraje favorablemente 24 de septiembre 2020 impuesta por la Supervisión y solo actúa como portavoz de la Secretaria de Educación Pública.

\section{Tabla 2. Resultados de la encuesta aplicada al personal docente}

\begin{tabular}{|c|c|c|c|c|c|c|c|c|}
\hline \multirow[b]{2}{*}{ Ítems } & Valor & Valor & \multicolumn{6}{|c|}{ Porcentaje Individual } \\
\hline & 5 & 1 & $\begin{array}{l}\text { Totalment } \\
\text { e en } \\
\text { desacuer } \\
\text { do } \\
(1)\end{array}$ & $\begin{array}{l}\text { En } \\
\text { desacuer } \\
\text { do } \\
\text { (2) }\end{array}$ & $\begin{array}{l}\text { De } \\
\text { acuerdo } \\
\text { (3) }\end{array}$ & $\begin{array}{c}\text { Muy de } \\
\text { Acuerd } \\
0 \\
(4)\end{array}$ & $\begin{array}{l}\text { Totalme } \\
\text { nte de } \\
\text { acuerdo } \\
\text { (5) }\end{array}$ & $\begin{array}{c}\text { Total, } \\
\text { porcenta } \\
\text { e }\end{array}$ \\
\hline $\begin{array}{l}\text { 1. Los maestros de esta escuela alcanzamos } \\
\text { acuerdos sobre objetivos y enfoques de programa } \\
\text { escuela de Tiempo Completo. }\end{array}$ & & & $0 \%$ & $0 \%$ & $58.3 \%$ & $41.7 \%$ & $0 \%$ & $100 \%$ \\
\hline $\begin{array}{l}\text { 2. Es una pérdida de tiempo hacer lo mejor } \\
\text { como profesor en esta escuela. }\end{array}$ & & & $0 \%$ & $100 \%$ & $0 \%$ & $0 \%$ & $0 \%$ & $100 \%$ \\
\hline $\begin{array}{l}\text { 3. Existe un gran esfuerzo colaborativo entre los } \\
\text { maestros de esta escuela para que los alumnos } \\
\text { aprendan. }\end{array}$ & & & $0 \%$ & $0 \%$ & $0 \%$ & $100 \%$ & $0 \%$ & $100 \%$ \\
\hline $\begin{array}{l}\text { 4. La administración de la escuela dedica parte } \\
\text { de su tiempo a ayudar a los profesores en sus } \\
\text { actividades escolares. }\end{array}$ & & & $41.7 \%$ & $58.3 \%$ & $0 \%$ & $0 \%$ & $0 \%$ & $100 \%$ \\
\hline $\begin{array}{l}\text { 5. Los maestros de esta escuela realizan su } \\
\text { trabajo con entusiasmo debido a que cuentan } \\
\text { con motivación de diversos tipos. }\end{array}$ & & & $0 \%$ & $91.7 \%$ & $8.3 \%$ & $0 \%$ & $0 \%$ & $100 \%$ \\
\hline $\begin{array}{l}\text { 6. Los maestros de esta escuela buscan otra } \\
\text { estrategia de enseñanza si sus estudiantes no } \\
\text { aprenden. }\end{array}$ & & & $0 \%$ & $0 \%$ & $66.7 \%$ & $33.3 \%$ & $0 \%$ & $100 \%$ \\
\hline $\begin{array}{l}\text { 7. En esta escuela, los maestros pueden hacer } \\
\text { poco para asegurar un alto nivel de logro en sus } \\
\text { alumnos. }\end{array}$ & & & $0 \%$ & $100 \%$ & $0 \%$ & $0 \%$ & $0 \%$ & $100 \%$ \\
\hline
\end{tabular}


"Fl suler ite mis hijos hard mi pronderis

Año 13.

Núm. 33
Revista de Investigación

Académica sin Frontera

ISSN: 2007-8870

\section{http://revistainvestigacionacademicasinfrontera.com}

Recibido el 2 de septiembre de 2020. Dictaminado mediante arbitraje favorablemente 24 de septiembre 2020

\begin{tabular}{|c|c|c|c|c|c|c|}
\hline $\begin{array}{l}\text { 8. Los maestros de esta escuela trabajamos } \\
\text { para crear unidades de aprendizaje que los } \\
\text { alumnos disfrutan aprendiendo en sus clases. }\end{array}$ & $0 \%$ & $0 \%$ & $100 \%$ & $0 \%$ & $0 \%$ & $100 \%$ \\
\hline $\begin{array}{l}\text { 9. Los maestros de esta escuela asesoran a los } \\
\text { alumnos que se les dificulta aprender, } \\
\text { apoyándolos fuera del horario de clases. }\end{array}$ & $0 \%$ & $100 \%$ & $0 \%$ & $0 \%$ & $0 \%$ & $100 \%$ \\
\hline $\begin{array}{l}\text { 10. Para los maestros de esta escuela es } \\
\text { responsabilidad proteger a los alumnos de la } \\
\text { deserción escolar. }\end{array}$ & $0 \%$ & $0 \%$ & $100 \%$ & $0 \%$ & $0 \%$ & $100 \%$ \\
\hline $\begin{array}{l}\text { 11. los maestros de esta escuela cuentan con } \\
\text { apoyo necesario que le permita realizar su } \\
\text { trabajo con calidad. }\end{array}$ & $0 \%$ & $100 \%$ & $0 \%$ & $0 \%$ & $0 \%$ & $100 \%$ \\
\hline 12. mi área de trabajo es motivante. & $100 \%$ & $0 \%$ & $0 \%$ & $0 \%$ & $0 \%$ & $100 \%$ \\
\hline $\begin{array}{l}\text { 13. La tarea del docente de esta escuela es } \\
\text { reconocida por los padres de familia. }\end{array}$ & $100 \%$ & $0 \%$ & $0 \%$ & $0 \%$ & $0 \%$ & $100 \%$ \\
\hline $\begin{array}{l}\text { 14. En la escuela se realizan acciones que } \\
\text { motivan, satisfacen y fortalecen las relaciones } \\
\text { humanas y el compromiso laboral. } \\
\text { 15.- Esta escuela se cuenta con la } \\
\text { infraestructura adecuada. }\end{array}$ & $100 \%$ & $91.7 \%$ & $0 \%$ & $8.3 \%$ & $0 \%$ & $100 \%$ \\
\hline $\begin{array}{l}\text { 16. Los conflictos que se presentan en la } \\
\text { escuela son resueltos de manera eficaz y } \\
\text { oportuna por la Dirección y el Personal } \\
\text { involucrado. }\end{array}$ & $0 \%$ & $25 \%$ & $75 \%$ & $0 \%$ & $0 \%$ & $100 \%$ \\
\hline $\begin{array}{l}\text { 17. Las autoridades educativas de esta escuela } \\
\text { tratan a los maestros como sus iguales. }\end{array}$ & $33.3 \%$ & $66.7 \%$ & $\%$ & $0 \%$ & $0 \%$ & $100 \%$ \\
\hline $\begin{array}{l}\text { 18. Las autoridades educativas de esta escuela } \\
\text { consultan a los maestros antes de tomar } \\
\text { decisiones que los afectan. }\end{array}$ & $0 \%$ & $83.3 \%$ & $16.7 \%$ & $0 \%$ & $0 \%$ & $100 \%$ \\
\hline $\begin{array}{l}\text { 19. El alto desempeño de las autoridades } \\
\text { educativas de esta escuela (compromiso con los } \\
\text { alumnos) es un ejemplo que impulsa a los } \\
\text { docentes a trabajar cada día mejor. }\end{array}$ & $8.3 \%$ & $90.7 \%$ & $0 \%$ & $0 \%$ & $0 \%$ & $100 \%$ \\
\hline $\begin{array}{l}\text { 20. Las autoridades educativas de esta escuela } \\
\text { ponen la muestra de dedicación al trabajo, al }\end{array}$ & $0 \%$ & $100 \%$ & $0 \%$ & $0 \%$ & $0 \%$ & $100 \%$ \\
\hline
\end{tabular}


Año 13.

Núm. 33
Académica sin Frontera

ISSN: 2007-8870

\section{http://revistainvestigacionacademicasinfrontera.com}

Recibido el 2 de septiembre de 2020. Dictaminado mediante arbitraje favorablemente 24 de septiembre 2020 disponer de tiempo e involucrarse en las actividades de la escuela.

21. Las autoridades educativas de esta escuela son consideradas por los maestros como una fuente de ideas para la mejora del servicio.

22. Los miembros de la autoridad educativa de la escuela son amigables y accesibles con todos los docentes.

23. La autoridad educativa de la escuela conoce los problemas que enfrentan los maestros y alumnos.

24. Los directivos de la escuela comunican a los maestros qué se espera de ellos.

25. Los profesores de esta escuela son atendidos por el Director cuando requieren de su ayuda.

26. La autoridad educativa de esta escuela está interesada en las innovaciones y en las nuevas ideas.

27. Los miembros de la escuela son reconocidos por las autoridades educativas de la escuela cuando hacen un buen trabajo.

28. El director está dispuesto hacer cambios para la mejorar el aprovechamiento escolar y la infraestructura de la escuela.

29. La responsabilidad de dirigir esta escuela es únicamente del director y no de otros miembros que lo apoyan en la dirección de la escuela.

30. Las autoridades educativas de esta escuela cumplen cabalmente con las funciones que le fueron asignadas y hacen cumplir a los docentes.

31. Las autoridades educativas de esta escuela fueron seleccionadas con base a los méritos requeridos para el puesto.

$\begin{array}{llllll}0 \% & 100 \% & 0 \% & 0 \% & 0 \% & 100 \%\end{array}$

$\begin{array}{llllll}0 \% & 100 \% & 0 \% & 0 \% & 0 \% & 100 \%\end{array}$
$8.3 \%$ $91.7 \%$ $0 \%$ $0 \%$

$100 \%$

$\mid$

$0 \%$

$0 \%$

$0 \%$

\begin{tabular}{|l|l}
\hline su & \\
&
\end{tabular}

$0 \%$ $66.7 \%$ $33.3 \%$ $0 \%$ $0 \%$

$100 \%$

\begin{tabular}{l|l|l|}
\hline & $0 \%$
\end{tabular}

(1)

$0 \%$

$100 \%$

$0 \%$

$0 \%$

$0 \% \quad 100 \%$

$33.3 \%$

$66.7 \%$

$0 \%$

$0 \%$

$0 \% \quad 100 \%$

-

es

$0 \%$

$83.3 \%$

$16.7 \%$

$0 \%$

$0 \% \quad 100 \%$

|

$33.3 \%$

$58.3 \%$

$8.3 \%$

$0 \%$

$0 \% \quad 100 \%$

a

|

$0 \%$

$75 \%$

$25 \%$

$66.7 \% \quad 33.3 \%$

$0 \%$

\begin{tabular}{lll}
$0 \%$ & $0 \%$ & $100 \%$ \\
& & \\
\hline 0 & $0 \%$ & $100 \%$ \\
\hline
\end{tabular}


Revista de Investigación

Año 13.

Académica sin Frontera

Núm. 33

ISSN: 2007-8870

http://revistainvestigacionacademicasinfrontera.com

Recibido el 2 de septiembre de 2020. Dictaminado mediante arbitraje favorablemente 24 de septiembre 2020

Nota: Esta Tabla enumera los ítems de motivación y liderazgo que fueron aplicados al personal de la institución, así como el valor que se les asigno a cada ítem en escala de Likert y el porcentaje de respuestas obtenidas en cada ítem.

Fuente: Elaboración propia

\section{CONCLUSIONES}

La tarea de enseñar requiere que el docente posea la adecuada formación y capacitación pedagógica correspondiente, de manera tal, que su labor e interacción con los alumnos resulte benéfica en ambos sentidos. El clima organizacional representa una problemática para la escuela objeto de estudio, ya que se percibe un clima afectado negativamente por la falta de motivación debido al tipo de liderazgo que prevalece en el ambiente. Según Goncalves (1999) define el clima organizacional como un fenómeno interviniente que media entre los factores del sistema organizacional y las tendencias motivacionales que se traducen en un comportamiento que tiene consecuencias sobre la organización. De este modo se demuestra la hipótesis planteada al inicio: la motivación y liderazgo son factores del clima organizacional, que afectan el grado de desempeño del personal docente que laboran la escuela primaria Miguel Hidalgo y Costilla del municipio de Centro, Tabasco. Las variables del clima organizacional definidas para este estudio, liderazgo y motivación, son factores que afectan el buen funcionamiento de la escuela primaria de la comunidad rural en cuestión, esto puede ser resultado de la forma en que se ha implementado el programa y a la débil gestión del directivo, ya que la institución no cuenta con los espacios adecuados para que los participantes se sientan motivados y comprometidos con la calidad educativa. El Programa de Escuela de Tiempo Completo (PETC) en el que se encuentra esta institución se implementó como apoyo para la mejora de calidad de la educación, sin embargo la calidad de la educación no se condiciona solo al aumento de horas en una jornada laboral. Diariamente se viven dentro del aula de 
"Fl suleer ide mis hiips

Año 13.

Núm. 33

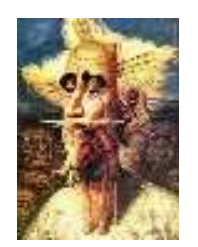

Revista de Investigación

Académica sin Frontera

ISSN: 2007-8870

\section{http://revistainvestigacionacademicasinfrontera.com}

Recibido el 2 de septiembre de 2020. Dictaminado mediante arbitraje favorablemente 24 de septiembre 2020 clases problemáticas tan relevantes como: la necesidad de pasar más tiempo en un aula de clases, concluir los libros de texto del alumno o cuando los aprendizajes no se alcanzan por cuestiones ligadas a limitaciones de la infraestructura, principalmente debido a las condiciones propias del clima tropical de la zona que genera temperaturas altas, la mayor parte del año. Los alumnos en su mayoría provienen de lugares apartados y carecen de aulas en buenas condiciones, en comparación con escuelas del área urbana. Estas últimas, son tomadas en cuenta por las autoridades para exhibirlas como resultados generados por el programa en curso. Para alcanzar cada uno de los objetivos que plantea la Secretaría de Educación Pública (SEP,2013), falta mejorar las condiciones de la infraestructura, ya que no es posible hablar de un Clima Organizacional adecuado, donde el ambiente tanto en infraestructura, como en organización no resulta confortable para los miembros del grupo.

El clima organizacional es afectado por el tipo de estructura impuesta por las autoridades educativas, estas no asumen el liderazgo. La plantilla laboral no es suficiente para cubrir puestos directivos, lo cual se convierte en una limitante ya que en muchas ocasiones los directores al mismo tiempo deben asumir el papel de docente frente a grupo y director de la escuela.

Por otra parte, hablar de motivación al mismo tiempo es hablar de liderazgo, dos variables básicas del clima organizacional, si son afectadas dentro de su ámbito, se perturban una a la otra, por tanto, ambas partes resultan afectadas.

Los docentes de esta institución educativa cumplen con cada una de sus actividades porque la responsabilidad la adquieren con sus educandos y no porque cuenten con estímulos que incrementen la motivación.

El clima organizacional de la institución es negativo e influido con bajas expectativas del personal que labora en la escuela, la motivación es poca y aunque existe el deseo de sobresalir y cumplir con los objetivos planteados, no son tomando en cuenta 0 
"Fl suler ide mis hiips

Año 13.

Núm. 33

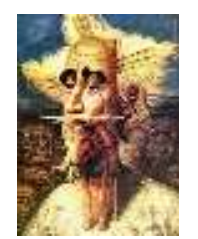

Revista de Investigación

Académica sin Frontera

ISSN: 2007-8870

\section{http://revistainvestigacionacademicasinfrontera.com}

Recibido el 2 de septiembre de 2020. Dictaminado mediante arbitraje favorablemente 24 de septiembre 2020 impulsados, ya que el liderazgo que existe es permisivo y se puede describir, como aquel que evita usar su poder y responsabilidad. El líder, aunque se preocupa por lo que está sucediendo es autocrático, ya que exige sin importar los medios que se usen para llegar a los resultados, porque es una institución que para cumplir, todos hacen una parte del trabajo, aunque no les corresponda son conscientes de los beneficios.

Los resultados de la investigación van más allá de los esperados, el clima organizacional de la institución se ve afectado en las variables, motivación y liderazgo. El hecho de afectar puede ser negativo o positivo y en esta investigación resulta negativo, al afectar los objetivos del personal que aun tratando de hacer lo mejor no cumplen con los estándares de calidad que la institución requiere. Existen otros factores que influyen en el clima organizacional como, la comunicación y las relaciones interpersonales, solo por mencionar algunos, sobre todo, las condiciones de la infraestructura que no han sido adecuadas para ser una institución generadora de conocimientos y de educación de calidad.

En razón del factor central que representan los alumnos, el personal docente y de apoyo, se integran algunas propuestas para intervenir en el proceso de cumplimiento de los objetivos planteados:

Para el ciclo escolar, se requiere apoyo para mejorar las condiciones de infraestructura de tal forma que el alumno y el docente se sientan motivados para llevar a cabo sus actividades. Así también motivar a los docentes, a través de capacitaciones que le permitan cumplir con los objetivos de la institución. El directivo debe auto diagnosticarse, revisar los patrones de liderazgo, lo cual le ayudarán a comprender el modo en que lleva a cabo las prácticas y procedimientos organizacionales, contribuyendo así a construir el tipo de liderazgo que beneficie el clima organizacional de la institución y se encauce hacia el logro de la anhelada educación de calidad. 
"Fl suler ise mis hijics

Año 13.

Núm. 33

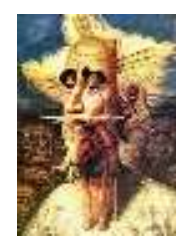

Revista de Investigación Académica sin Frontera ISSN: 2007-8870

http://revistainvestigacionacademicasinfrontera.com

Recibido el 2 de septiembre de 2020. Dictaminado mediante arbitraje favorablemente 24 de septiembre 2020 REFERENCIAS BIBLIOGRÁFICAS

Amorós, E. (2007). Comportamiento Organizacional: En Busca del Desarrollo de Ventajas Competitivas. Escuela de Economía USAT. Lambayeque, Perú.

Arce, A. (2017) Curso de Actualización en Gestión del Clima Organizacional. Perú: Pontificia Universidad Católica del Perú, curso Clima Organizacional.

Arias, F. (2006). El proyecto de investigación. Editorial episteme. Caracas.

Chiavenato, I (2002). Administración en los nuevos tiempos. Bogota: Mcgraw-hill.

Goncalves, A. (1999). Dimensiones del clima organizacional. Recuperado de https://bit.ly/2CmuNQF

Hernández, R., Fernández, C. y Baptista, P. (2010). Metodología de la Investigación. México: McGraw-Hill

Lamoyi, C. L. (2007). La organización social de la escuela. México: Compañía Editorial Impresa.

Lincoln, Y. y Guba, E. G. (2000). Paradigmatic controversies, contradictions and emerging confluences. En N. Denzin y Y. Lincoln. Thousand Oaks, Sage, CA, Estados Unidos: Handbook of Qualitative Research.

Martínez, M. (2003). La gestión empresarial: equilibrando objetivos y valores. Madrid: Díaz de Santos. 
"Fl suler ise mis hijics

Año 13.

Núm. 33

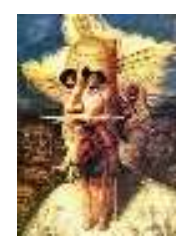

Revista de Investigación Académica sin Frontera ISSN: 2007-8870

$\underline{\text { http://revistainvestigacionacademicasinfrontera.com }}$

Recibido el 2 de septiembre de 2020. Dictaminado mediante arbitraje favorablemente 24 de septiembre 2020

Maslow, A. H. (1991). Motivación y personalidad. Ediciones Díaz de Santos, S.A. Madrid, España.

Medina, A. (2017). Incidencia del clima organizacional en el desempeño docente de la Unidad Educativa Particular Santo Domingo de Guzmán - Quito. Quito, Ecuador: Universidad Andina Simón Bolívar. Recuperado de https://bit.ly/2DakyRo

Montalván, C. (1999). Los recursos en la pequeña y mediana empresa. México: Universidad Iberoamericana, Dirección de Difusión Universitaria.

SEP (Secretaría de Educación Pública). (2013). Censo de Escuelas, Maestros y Alumnos de Educación Básica y Especial. Recuperado de https://bit.ly/2RvuCwU

SEP (Secretaría de Educación Pública). (2014). Programa Escuelas de Tiempo Completo. Recuperado de https://bit.ly/2KKoCZb

Van der Westhuizen, P.C. y Mentz, P. J. (2007). An ontological perspective on the school as an organization. Schools as organisations. Van Schaik Publishers. 3 rd edition,4th impression. Hatfield, South Africa.

Vélaz de Medrano, C., y Vaillant, D. (2009). Aprendizaje y desarrollo profesional docente. Madrid, España: Fundación Santillana. Recuperado de https://bit.ly/1SDDjhA 
"Fl solber ile mis hiips

Año 13.

Núm. 33
Revista de Investigación

Académica sin Frontera

ISSN: 2007-8870

\section{http://revistainvestigacionacademicasinfrontera.com}

Recibido el 2 de septiembre de 2020. Dictaminado mediante arbitraje favorablemente 24 de septiembre 2020

\section{Directorio Institucional}

Dr. Enrique Fernando Velázquez Contreras

Rector

Dr. Ramón Enrique Robles Zepeda

Secretario General Académico

Dra. Rosa María Montesinos Cisneros

Secretaria General Administrativa

Dr. Rodolfo Basurto Álvarez

Director de Vinculación y Difusión

Dra. Adriana Leticia Navarro Verdugo

Vicerrectora de la Unidad Regional Sur

Dr. Ernesto Clark Valenzuela

Director de la División de Ciencias Económicas y Sociales

Dr. Francisco Espinoza Morales

Secretario de la División de Ciencias Económico y Sociales

Dra. Leticia María González Velásquez

Jefe del Departamento de Ciencias Económico Administrativas

Dra. Lidia Amalia Zallas Esquer

Jefe de Departamento de Ciencias Sociales 
"Fl suler ide mis hiipses hard mi pronderis

Año 13.

Núm. 33

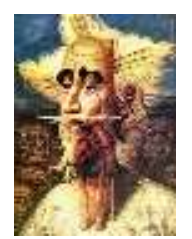

Revista de Investigación

Académica sin Frontera

ISSN: 2007-8870

\section{http://revistainvestigacionacademicasinfrontera.com}

Recibido el 2 de septiembre de 2020. Dictaminado mediante arbitraje favorablemente 24 de septiembre 2020

\section{Comité Directivo}

\section{Editor Responsable}

Dr. Francisco Espinoza Morales

\section{Directora}

Dra. Leticia María González Velásquez

Subdirector

Dr. Javier Carreón Guillen

\section{Editor Científico}

Dr. Cruz García Lirios

\section{Master Gráfico}

M.T.I. Francisco Alan Espinoza Zallas

Nos complace anunciar que su diario, "Academic Research Journal Withoutborders" (ISSN/EISSN 20078870) fue evaluado positivamente en la indexación Citefactor, ahora la página de la revista está disponible en línea, en caso de cualquier problema.

Journals Master | International Innovative Journal Impact Factor (IIJIF)

\section{Red Latinoamericana de revistas Académicas en Ciencias Sociales y Humanidades}
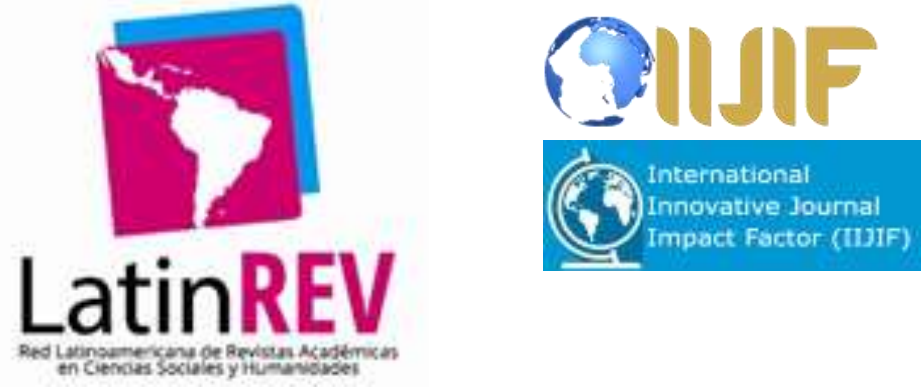
Núm. 33

ISSN: 2007-8870

\section{http://revistainvestigacionacademicasinfrontera.com}

Recibido el 2 de septiembre de 2020. Dictaminado mediante arbitraje favorablemente 24 de septiembre 2020

\section{Comité editorial}

Dra. Angélica María Rascón Larios

Universidad de Sonora. México

Dra. María del Rosario Molina González

Universidad de Sonora

Dra. Francisca Elena Rochin Wong

Universidad de Sonora. México

Dra. Lidia Amalia Zallas Esquer

Universidad de Sonora. México

Dra. Beatriz Llamas Arechiga

Universidad de Sonora. México

Dr. Rogelio Barba Álvarez

Universidad de Guadalajara. México

Dra. Rosa María Rincón Ornelas

Universidad de Sonora. México

Dr. Juan Flores Preciado

Universidad de Colima. México

Dr. Amado Olivares Leal. Universidad de Sonora

Universidad de Sonora. México

Dr. Guillermo Velázquez Valadez.

Instituto Politécnico Nacional (IPN) México

Dr. Hugo Nefstalí Padilla Torres.

Universidad Estatal de Sonora. México

Dr. Luis Ramón Moreno Moreno.

Universidad Autónoma de Baja California. México

Dr. Miguel Ángel Vázquez Ruiz.

Universidad de Sonora. México 
Núm. 33

ISSN: 2007-8870

\section{http://revistainvestigacionacademicasinfrontera.com}

Recibido el 2 de septiembre de 2020. Dictaminado mediante arbitraje favorablemente 24 de septiembre 2020

Dra. Lorena Vélez García.

Universidad Autónoma de Baja California. México

Dra. Pabla Peralta Miranda.

Universidad Simón Bolívar, Barranquilla, Colombia

Mtro. Roberto Espíritu Olmos

Universidad de Colima (FCA Tecomán) Colima

Dr. Héctor Priego Huertas.

Universidad de Colima (FCA Tecomán) Colima

Mtra. María Guadalupe Alvarado Ibarra.

Universidad de Sonora. México.

MSc. Celso Germán Sánchez Zayas

Universidad de Camagüey, Ignacio Agramonte Loynaz, Cuba

Dra. María Luisa Quintero Soto

Universidad Autónoma del Estado de México

Dr. Eyder Bolivar Mojica

Universidad Católica, Luis Amigó, Medellin, Colombia

Revisores de Textos en Inglés

Mtro. Renato Encinas

Mtra. Cecilia Guadalupe Martínez Solano

\section{Comité científico}

Dr. Rosendo Martínez Jiménez. Universidad Autónoma Benito Juárez de Oaxaca.

Dr. Hugo Neftalí Padilla. Universidad Estatal de Sonora

Dra. María Teresa Gaxiola Sánchez. Universidad de Sonora.

Dr. José Cesar Kaplan. Universidad Estatal de Sonora.

Dr. Alfredo Islas Rodríguez. Universidad de Sonora

Frecuencia de publicación: semestral / 2 números por año. 


\section{http://revistainvestigacionacademicasinfrontera.com}

Recibido el 2 de septiembre de 2020. Dictaminado mediante arbitraje favorablemente 24 de septiembre 2020

Revista de Investigación Académica sin Frontera (RIASF) con (ISSN: 2007-8870) es un interlocutor internacional de acceso abierto revisado diario en línea en el ámbito del de las Ciencias Económicas Administrativas y Sociales. Su objetivo principal es dar a los trabajos de investigación de calidad. Cubre todas las sub-campos de los campos anteriormente mencionados. Proporciona la plataforma a académicos, estudiantes y profesionales. Sólo pública trabajos de investigación y artículos de revisión inicial. Documento presentado debe cumplir con algunos criterios como, debe ser original, inédita y no estén sometidos a ninguna otra revista. RIASF es una revista arbitrada / Revisión por pares International. Publicamos documentos sobre una variedad de temas, contextos y estrategias de análisis que examinan la relación entre la rápida evolución para la Sociedad y la tecnología del conocimiento.

REVISTA DE INVESTIGACIÓN ACADÉMICA SIN FRONTERA, Año 13, No. 33, Julio - diciembre 2020, es una publicación semestral de investigación científica, editada por la Universidad de Sonora, a través de las División de Ciencias Económicas y Sociales, de la Unidad Regional Sur, Blvd. Lázaro Cárdenas No. 100, Col. Francisco Villa, Navojoa, Sonora, Sonora, México, C.P. 85880. Tel. (642) 425- 99-54.

http://www.revistainvestigacionacademicasinfrontera.com/, revistaacademicasinfrontera@ unison.mx.

Editor responsable: Francisco Espinoza Morales. Reserva de Derechos al Uso Exclusivo: 04-2013121811323700-203 e ISSN: 2007-8870, ambos otorgados por el Instituto Nacional de Derecho de Autor. Inscrita en el Directorio de LATINDEX, con Núm. De folio 20014, folio único 14590. Responsable de la última actualización de este Número, Unidad Informática de la Universidad de Sonora, fecha de la última modificación, 30 de diciembre 2020, indexada a Cite Factor Academic Scientific Journal y Journals Master (IIJIF) y Red Latinoamericana de Revistas Académicas en Ciencias Sociales y Humanidades, (Latín Rev). Las opiniones expresadas por los autores no necesariamente reflejan la postura del editor de la publicación. Se autoriza la reproducción total o parcial de los contenidos e imágenes en la presente publicación siempre y cuando se cuente con la autorización del editor y se cite plenamente la fuente.

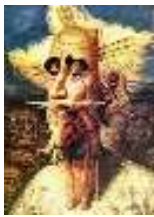


"Flis suber ile mis hiipses hará mi prondiogic

Año 13.

Núm. 33

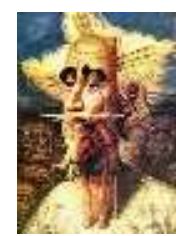

Revista de Investigación

Académica sin Frontera

ISSN: 2007-8870

\section{http://revistainvestigacionacademicasinfrontera.com}

Recibido el 2 de septiembre de 2020. Dictaminado mediante arbitraje favorablemente 24 de septiembre 2020

Nos complace anunciar que su diario, "Academic Research Journal Withoutborders" (ISSN/EISSN 20078870) fue evaluado positivamente en la indexación Citefactor, ahora la página de la revista está disponible en línea, en caso de cualquier problema.

Journals Master | International Innovative Journal Impact Factor (IIJIF)

\section{Red Latinoamericana de revistas Académicas en Ciencias Sociales y Humanidades}

\section{CiteFactor}
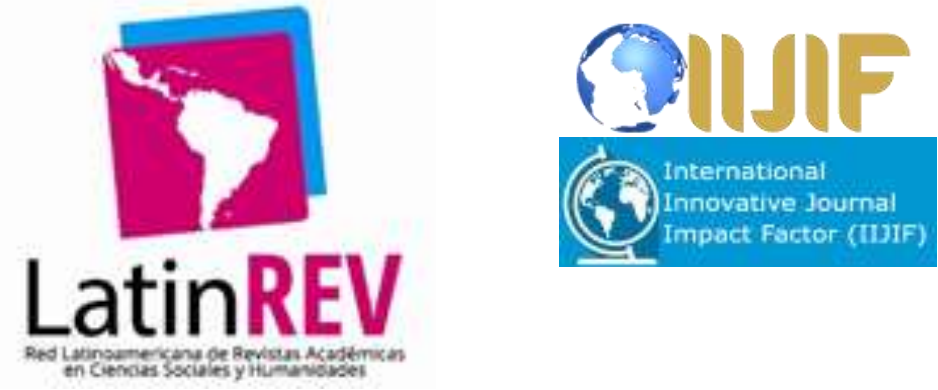

https://www.neliti.com

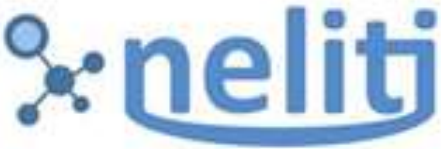

Indonesia's Research Repository

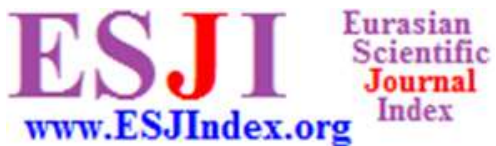

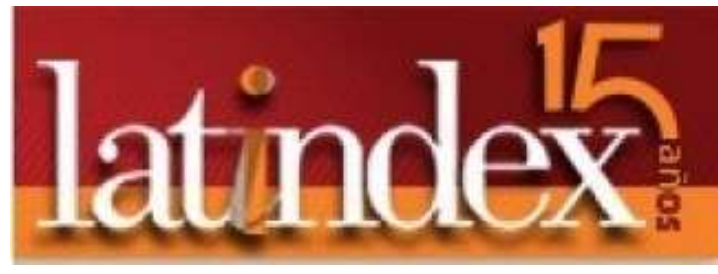

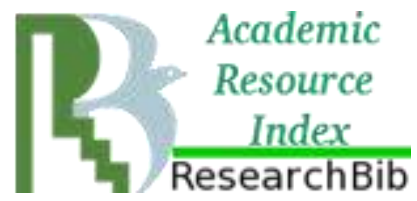


Revista de Investigación

Año 13.

Académica sin Frontera

Núm. 33

ISSN: 2007-8870

$\underline{\text { http://revistainvestigacionacademicasinfrontera.com }}$

Recibido el 2 de septiembre de 2020. Dictaminado mediante arbitraje favorablemente 24 de septiembre 2020 